\title{
Security Margin Assessment Method of Centralized Spot Market Based on Information Entropy
}

\author{
Leng Yue ${ }^{1}$, Li Lili ${ }^{2}$, Zhang Feng ${ }^{3}$, Zhou Ziqing ${ }^{4}$, Wang Zhiqi ${ }^{5 *}$, Wu Shuomin ${ }^{6}$ \\ ${ }^{1}$ Nari Group Corporation(State Grid Electric Power Research Institute), State Key Laboratory of Smart Grid Protection and Control, \\ Nanjing, China \\ ${ }^{2}$ Nari Group Corporation(State Grid Electric Power Research Institute), State Key Laboratory of Smart Grid Protection and Control, \\ Nanjing, China \\ ${ }^{3}$ State Grid Zhejiang Electric Power CO., LTD. Research Institute, State Grid Zhejiang Electric Power CO., LTD. Power Market Simulation \\ Laboratory, Hangzhou, China \\ ${ }^{4}$ State Grid Zhejiang Electric Power CO., LTD. Research Institute, State Grid Zhejiang Electric Power CO., LTD. Power Market Simulation \\ Laboratory, Hangzhou, China \\ ${ }^{5}$ Nari Group Corporation(State Grid Electric Power Research Institute), State Key Laboratory of Smart Grid Protection and Control, \\ Nanjing, China \\ ${ }^{6}$ Nari Group Corporation(State Grid Electric Power Research Institute), State Key Laboratory of Smart Grid Protection and Control, \\ Nanjing, China
}

\begin{abstract}
In the centralized spot market, each participant pursues the maximization of economic benefits, which leads to the operation condition of the power grid closer to the security boundary. Thus, in this paper, a novel approach to security margin assessment of the centralized spot market is proposed. By proceeding from the mechanism of influence of load variation on power flow, the relationship between power flow transfer characteristics and the security margin of the power grid is explored. Then, on the basis of information entropy theory, the power flow transfer balancing evaluation index of the power grid is established. And this index is applied for security margin assessment of the power grid in the environment of the centralized spot market. Results of an IEEE 14-bus system case study have validated the effectiveness and quickness of the proposed method. Meanwhile, in accordance with the analysis results, it is proved that the proposed method is capable of accurately identifying the critical power transmission and transformation equipment affecting the security margin of the power grid, which is helpful to identify the potential risks of the operation of the power grid in advance.
\end{abstract}

\section{INTRODUCTION}

The operation of the centralized spot market will have an extensive and profound impact on the whole power industry [1]. With the implementation of the reform scheme of the power system in China, the economy plays a more and more important role in the operation of the power grid. Thus, the generation dispatching plan in the spot market mode no longer conforms to the balanced distribution, and the power flow distribution in the spot market mode is also different from that in planned dispatching mode [2]. As a result, the uncertainty of power flow distribution brought by the centralized spot market will bring new challenges to the safe operation of the power grid [3]. Therefore, the traditional analysis method of the safe operation of the power grid cannot fully meet the needs of the development of the power grid in the centralized spot market mode. At present, the relevant research results on the centralized spot market at home and abroad mostly focus on the market rules [4], market

* Corresponding author: 772578594@qq.com schemes [5], market construction paths [6], marketclearing methods et al. [7]. Nevertheless, it can be seen that there is a lack of necessary research and application on power flow transfer characteristics and security margin assessment of power grid in centralized spot market mode.

As an effective supplement to the traditional analysis method of safe operation of the power grid, many research achievements have been made in power flow balancing analysis of the power grid [8-11]. Taking the load factor of the line as the assessment object, the application of line load balancing in severity evaluation of power flow overrun, identification of dangerous lines, and judgment of the self-organized critical state of the power system are analyzed respectively in [8-10]. The expected value of the blackout sequence of the power system is reduced by studying power flow balancing between regions in [11]. This kind of method mentioned above is based on the known operation status of the power grid, without considering the transfer of its power flow. Given the above situation, aiming at the variability of power grid operation mode in the environment of the power market, this paper adopts the power flow transfer balancing evaluation index 
to describe the equilibrium level of power flow transfer when the operation mode of the power grid changes.

As is known to all, the security margin of the power grid is related not only to its structure but also to its operation mode. Specifically, in different operation modes, the distribution characteristics of power flow transfer of the power grid are different, as well as the ability to withstand load variation. In the case that each branch undertakes the power flow transfer according to its capacity margin, the power grid has a strong ability to withstand load variation and a large security margin. Hence, the power flow transfer balancing evaluation index is constructed to assess the security margin of the power grid in this paper.

Nowadays, entropy theory is a new method and idea for analyzing the operation characteristics of the modern complex large power grid, which has been applied in many fields of power system, such as reliability assessment [12], available transmission capacity assessment [13], optimal power flow calculation [14], probabilistic power flow modeling [15], fault detection et al. [16]. It is well known that entropy can describe the characteristics of power flow. Based on the analysis of the operation characteristics of the power grid, the power flow entropy index is constructed to describe the operation characteristics of the power grid, such as the power flow entropy index constructed in [17].

In this paper, the power flow transfer balancing evaluation index is established based on entropy theory to analyze the equilibrium level of power flow transfer caused by load variation, and the index is applied to assess the security margin of the power grid in the centralized spot market mode.

\section{PoWer flow tRANSfER BALANCING}

\subsection{Power Flow Balancing}

Power flow transfer balancing describes the equilibrium level of power flow transfer when the operation mode and load variation mode of the power grid both are known, which can take into account the operation characteristics of the power grid and the influence of load variation at the same time. Specifically, the more balanced the change of branch power caused by load variation, the more balanced each branch bears the change of load power, and it is not easy to cause branch out of limit and affect the safe operation of the power grid.

\subsection{Information Entropy}

As an effective method to quantify the irregularity, information entropy can be used to quantitatively describe the equilibrium level of power flow transfer. Therefore, the characteristic quantity of power flow transfer with load variation can be extracted, and the power flow transfer balancing evaluation index based on entropy theory can be established.

Information entropy is defined as follows [18]:

$$
H=-C \sum_{k=1}^{N} P(k) \ln P(k)
$$

where $H$ indicates the information entropy. $C$ is the constant. $N$ represents the total number of events. $P(k)$ stands for the percentage of the $k^{\text {th }}$ value $x_{k}$ in the sum of all values of this physical quantity, which is given by

$$
P(k)=\frac{x_{k}}{\sum_{i=k}^{N} x_{k}}
$$

The larger the entropy, the better the equilibrium level of power flow transfer. On the contrary, the worse the equilibrium level of power flow transfer.

\section{POWER FLOW TRANSFER BALANCING EVALUATION INDEX BASED ON INFORMATION ENTROPY}

In general, information entropy can represent the equilibrium level of power flow transfer [19]. Combining the information entropy theory with the characteristics of power flow transfer with load variation, the power flow transfer balancing evaluation index is constructed as follows.

In the current operation mode, each branch of the power grid has a certain power margin. Power margin of branch $i$ is described as

$$
P_{i}^{m}=P_{i}^{\max } \pm\left|P_{i}^{0}\right|
$$

where $P_{i}^{\max }$ acts as the upper power limit of branch $i . P_{i}^{0}$ indicates the power of branch $i$ in the current operation mode.

The vector of branch power margin made up of $P_{i}^{m}$ can be constructed as

$$
\boldsymbol{P}^{m}=\left[P_{1}^{m}, P_{2}^{m}, \ldots, P_{i}^{m}, \ldots, P_{N}^{m}\right]
$$

where $N$ denotes the total number of branches.

The variation of branch power caused by unit load variation is expressed as the vector $\boldsymbol{\Delta P}$ :

$$
\Delta \boldsymbol{P}=\left[\Delta P_{1}, \Delta P_{2}, \ldots, \Delta P_{i}, \ldots, \Delta P_{N}\right]
$$

The power variation of branch $i$ is calculated as

$$
\Delta P_{i}=\sum_{k=1}^{M} D_{i k} \Delta P_{k}
$$

The power flow distribution factor of branch $i$ relative to node $k$ can be then derived as follows:

$$
D_{i k}=\frac{X_{m k}-X_{n k}}{x_{i}}
$$


where $\triangle P_{k}$ represents the load variation of node $k$. The head and end nodes of branch $i$ are $m$ and $n$ respectively. $X_{m k}$ and $X_{n k}$ are deemed to be the corresponding elements in the impedance matrix of network nodes. $x_{i}$ stands for the reactance of branch $i$.

In the case of unit variable load, the power flow transfer rate describing the power increment of branch $i$ relative to the power margin of this branch is computed as

$$
P_{i}^{t}=\frac{\Delta P_{i}}{P_{i}^{m}}
$$

The vector of branch power flow transfer rate composed of $P_{i}^{t}$ is constructed as follows:

$$
\boldsymbol{P}^{t}=\left[P_{1}^{t}, P_{2}^{t}, \ldots, P_{i}^{t}, \ldots, P_{N}^{t}\right]
$$

The standardized vector $\boldsymbol{P}$ can be formed as

$$
\boldsymbol{P}=[P(1), P(2), \ldots, P(i), \ldots, P(N)]
$$

where $P(i)$ is the ratio of the power flow transfer rate of branch $i$ in the sum of the power flow transfer rates of all branches, which can be expressed as

$$
P(i)=\frac{P_{i}^{t}}{\sum_{i=1}^{N} P_{i}^{t}}
$$

Substituting $P(i)$ into equation (1), the power flow transfer entropy can be obtained as follows:

$$
H=-\frac{1}{\ln \mathrm{N}} \sum_{i=1}^{N} P(i) \ln P(i)
$$

Obviously, the power flow transfer entropy is constructed to assess the power flow transfer balancing of the power grid. It can be verified that the larger the entropy, the more balanced the distribution of power flow transfer on each branch caused by load variation, meanwhile, the power grid has a stronger ability to withstand the load variation. Conversely, the ability of the power grid to sustain the load variation is weak.

\section{SECURITY MARGIN ASSESSMENT OF THE POWER GRID BASED ON POWER FLOW TRANSFER ENTROPY}

In general, the repeated power flow method $[20,21]$ can be applied to calculate the security margin of the power grid in a certain operation mode. However, the operation condition of the power grid changes in real-time, in which the adjustment of generator output, the change of load, the switch of equipment operating condition, and so on will affect the security margin of the power grid. Besides, the calculation speed of the repeated power flow method is far from meeting the online requirements for the large-scale power grid. Therefore, the repeated power flow method is widely used in offline calculation [20]. Hence, it is of great significance to explore a fast and reliable index to replace the repeated power flow method to describe the security margin of the power grid in different operation modes.

The security margin of the power grid in different operation modes is different, which is related to the equilibrium level of the distribution of branch power variation caused by the load variation in this operation mode. The more balanced the branch power variation caused by the load variation, the more balanced each branch withstands load variation so that the power grid has a greater security margin in this operation mode. On the contrary, the more unbalanced the branch power variation caused by load variation, the lower the security margin of the power grid in this operation mode. Therefore, this paper applies the power flow transfer entropy index to assess the security margin of the power grid.

In several operation modes, the security margins of the power grid are calculated by repeated power flow method, and the power flow transfer entropy values are calculated by using the method proposed above. The calculation results are shown in Fig. 1.

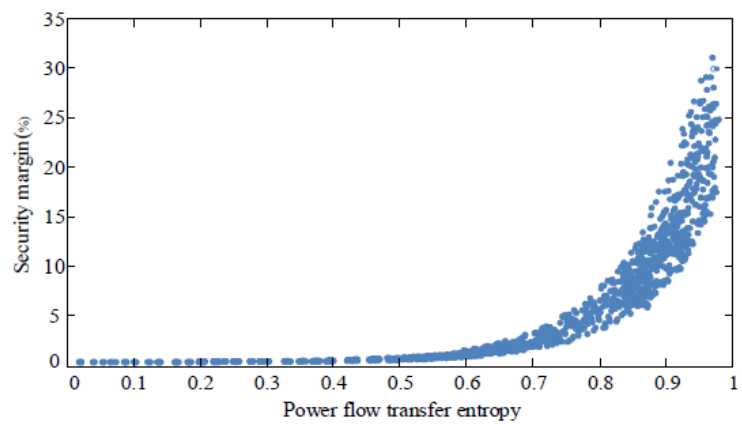

Figure 1. Relationship between power flow transfer entropy and security margin.

Each point in Fig. 1 represents an operation mode of the power grid. As can be seen from Fig. 1, with the increase of power flow transfer entropy, the security margin of the power grid increases. When the power flow transfer entropy is less than a certain value, the security margin remains at a low level. Otherwise, when the power flow transfer entropy is larger, the safety margin is also larger. It is illustrated that the power flow transfer entropy index can reflect the security margin level of the power grid, which verifies the effectiveness of the index proposed in this paper.

In terms of calculation speed, for a 159-bus power grid, it takes $0.041 \mathrm{~s}$ to calculate the power flow transfer entropy in a specific operation mode, while the time to calculate the security margin in the same operation mode by using the repeated power flow method is usually more than seconds. The above analysis shows that the method presented in this paper has high computational efficiency and is suitable for online security assessment.

\section{EXAMPLE CALCULATION}

Based on the IEEE 14-bus system shown in Fig. 2, an example is constructed to further verify the research 
content of this paper. In the example, the bidding method of generators adopts the segmented bidding method, and the data are shown in Table I. Also, the load variation mode is assumed to be uniform, that is, the load power of each load node changes in an equal proportion. Specifically, three scenarios are constructed for calculation, and the data are shown in Table II. The repeated power flow method is used as the comparison algorithm to verify the effectiveness of the power flow transfer entropy index described in this paper.

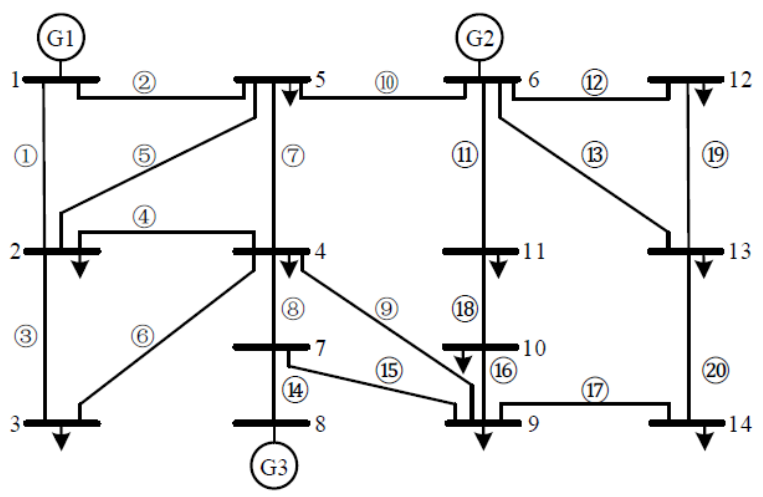

Figure 2. IEEE 14-bus system.

TABLE I. GENERATOR OFFER PRICE BY OUTPUT

\begin{tabular}{ccccc}
\hline Generator & Segment & $\begin{array}{c}\text { Initial } \\
\text { capacity } \\
\text { (MW) }\end{array}$ & $\begin{array}{c}\text { Termination } \\
\text { capacity } \\
\text { (MW) }\end{array}$ & $\begin{array}{c}\text { Price } \\
\text { (RMB/MWh) }\end{array}$ \\
\hline \multirow{3}{*}{ G1 } & 1 & 0.00 & 150.00 & 190.00 \\
& 2 & 150.00 & 200.00 & 240.00 \\
& 3 & 200.00 & 250.00 & 290.00 \\
& 4 & 250.00 & 300.00 & 320.00 \\
G2 & 5 & 300.00 & 330.00 & 390.00 \\
\hline \multirow{3}{*}{ G3 } & 1 & 0.00 & 100.00 & 220.00 \\
& 2 & 100.00 & 120.00 & 260.00 \\
& 3 & 120.00 & 150.00 & 310.00 \\
\hline
\end{tabular}

TABLE II. COMPUTING SCENARIOS

\begin{tabular}{cc}
\hline Scenario & Transmission power limits of branches \\
\hline 1 & The transmission power limit of branch (7) is \\
250MW & The transmission power limit of branch (7) is \\
$150 \mathrm{MW}$ & The transmission power limit of branch (7) is \\
& 150MW, and that of branch (9) is 100MW \\
\end{tabular}

According to the above data, the power flow transfer entropy and the corresponding power flow transfer rates of each branch of the three scenarios are calculated respectively, for the purpose of demonstrating that the power flow transfer entropy can reflect the equilibrium level of power flow transfer of the branch caused by load variation. The calculated results are displayed in Table III and Fig. 3.
TABLE III. CALCULATION RESUlTS OF POWER FLOW TRANSFER ENTROPY

\begin{tabular}{ccc}
\hline Scenario & Power flow transfer entropy & Security margin $/ \%$ \\
\hline 1 & 0.81 & 62.4 \\
2 & 0.65 & 37.9 \\
3 & 0.49 & 12.7 \\
\hline
\end{tabular}

It can be seen from Table III that in scenario 1, the power flow transfer entropy is 0.81 and the security margin is $62.4 \%$; in scenario 2 , the power flow transfer entropy is 0.65 and the security margin is $37.9 \%$; in scenario 3 , the power flow transfer entropy is 0.49 and the security margin is $12.7 \%$. Thus, the power flow transfer entropy index constructed in this paper can accurately reflect the level of power grid security margin.

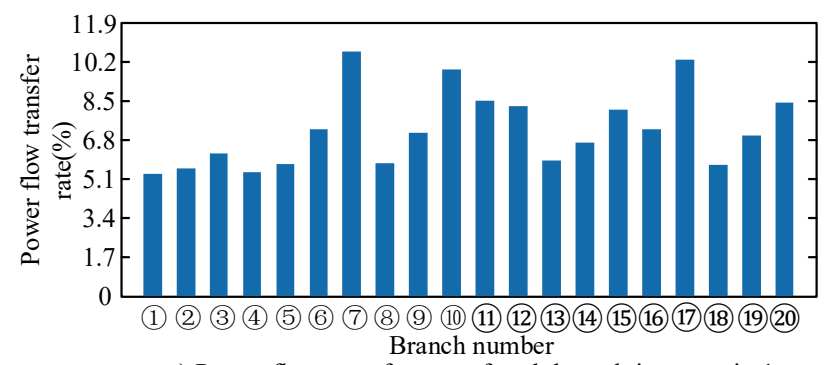

a) Power flow transfer rate of each branch in scenario 1

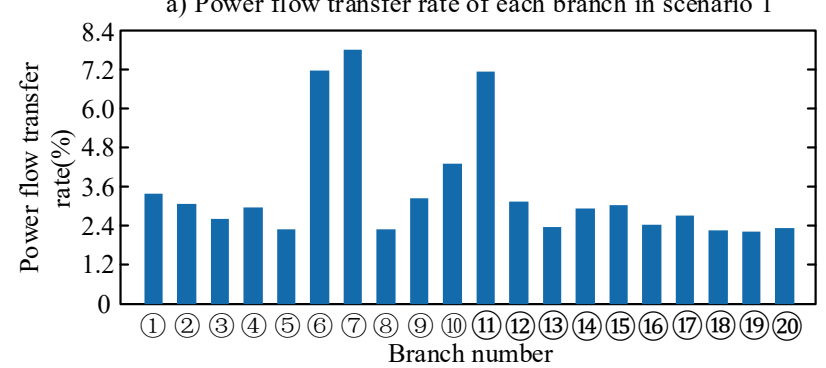

b) Power flow transfer rate of each branch in scenario 2

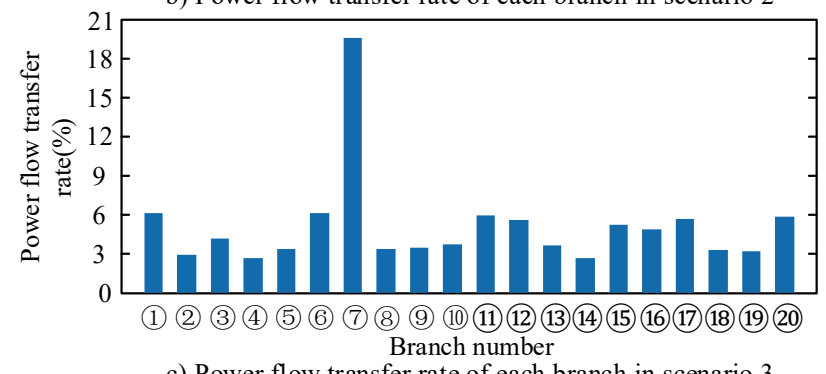

c) Power flow transfer rate of each branch in scenario 3

Figure 3. Power flow transfer rate of each branch.

As you can see from Fig. 3 that with the decrease of power flow transfer entropy, the distribution of the power flow transfer rate of each branch becomes more and more unbalanced. In scenario 3, the power flow transfer entropy of the power grid is small, and the power flow variation of each branch is extremely unbalanced, while that of other branches is very small. In this case, the branch with the largest power flow transfer rate will become the key branch to limit the security margin of the power grid.

From all the aforementioned analysis, it can be perceived that the power flow transfer entropy index proposed in this paper can effectively reflect the equilibrium level of the power flow transfer rate of each 
branch, and can effectively recognize the key branches that affect the security margin of the power grid.

\section{Conclusions}

In this paper, based on the mechanism of the influence of load variation on the branch power flow, the power flow transfer entropy index is established and its effectiveness is verified. On this basis, the index is applied to assess the security margin of the power grid in the centralized spot market mode. Furthermore, in light of the comparative analysis of different scenarios with different branch transmission power limits, it is observed that the power flow transfer entropy index constructed in this paper can accurately reflect the security margin level of the power grid, and can effectively identify the key branches that affect the security margin of the power grid. Moreover, it is very helpful for dispatchers to identify the potential risks of the operation of the power grid in advance and ensure the long-term safe and stable operation of the power grid.

\section{ACKNOWLEDGMENTS}

This work is supported by State Grid Corporation of China (No. 1300-202055035A-0-0-00).

\section{REFERENCES}

1. GAO Zhiyuan, FENG Shuhai, XU Lizhong, et al. Business Framework Design of Provincial Dispatching Center in Centralized Electricity Spot Market[J]. Automation of Electric Power Systems, 2019, 43(18): 185-191.

2. CAO Weibin. Study on Reserve Procurement and Pricing Considering Key Security Constraints[D]. Shanghai: Shanghai Jiao Tong University, 2010.

3. JIAN Hongyu, KANG Chongqing, ZHONG Jin, et al. Research on Identification of Operation State in Electricity Market[J]. Proceedings of the CSEE, 2007, 27(22): 63-68.

4. SONG Yonghua, BAO Minglei, DING Yi, et al. Review of Chinese Electricity Spot Market Key Issues and Its Suggestions Under the New Round of Chinese Power System Reform[J]. Proceedings of the CSEE, 2020, 40(10): 128-143.

5. Wan Chulin. Analysis of Foreign Power Market and Design of Southern Region Power Market[D]. Guangzhou: South China University of Technology, 2018.

6. Ge Rui, Chen Longxiang, Wang Yiyu, et al. Optimization and Design of Construction Route for Electricity Market in China[J]. Automation of Electric Power Systems, 2017, 41(24): 10-15.

7. Sun $X$, Luh $\mathrm{P}$, Bragin $\mathrm{M}$, et al. A Novel Decomposition and Coordination Approach for Large Day-Ahead Unit Commitment With Combined Cycle
Units[J]. IEEE Transactions on Power Systems, 2018, 33(5): 5297-5308.

8. LOU Suhua, WU Yaowu, LI Tingting, et al. The Characteristics Analysis of Improving Power Grid Balance by ESS Allocated in Demand Side[J]. Journal of Electric Power Science and Technology, 2013, 28(3): 39-44.

9. WU Yaowu, CAI Zhijing, LOU Suhua, et al. Transmission Network Expansion Planning Model Considering Network Load Balance[J]. Journal of Huazhong University of Science and Technology(Natural Science Edition), 2013, 41(12): 96-100.

10. CAO Yijia, WANG Guangzeng, CAO Lihua, et al. An Identification Model for Self-organized Criticality of Power Grids Based on Power Flow Entropy[J]. Automation of Electric Power Systems, 2011, 35(7): 1-6.

11. HE Qing, GUO Jianbo. Balance Control for Decreasing Expected Value of Blackout Sequence[J]. Proceedings of the CSEE, 2012, 32(4): 65-69.

12. JIANG Qun. Evaluation of Power System Reliability Magin[D]. Beijing: North China Electric Power University, 2011.

13. ZHENG Yanan, ZHOU Ming, LI Gengyin. An Information Entropy-Based Contingency Enumeration Approach for Available Transfer Capability Assessment[J]. Power System Technology, 2011, 35(11): 107-113.

14. LI Zhiming, LI Wweixing, WANG Yongjian. Surrogate Constraint Algorithm for Optimal Power Flow Based on Entropy Theory[J]. Automation of Electric Power Systems, 2001, 25(11): 28-31.

15. WILLIAMS T, CRAWFORD C. Probabilistic Load Flow Modeling Comparing Maximum Entropy and Gram-Charlier Probability Density Function Reconstruction[J]. IEEE Trans on Power Systems, 2013, 28(1): 272-280.

16. HE Zhengyou, CAI Yumei, QIAN Qingquan. A Study of Wavelet Entropy Theory and Its Application in Electric Power System Fault Detection[J]. Proceedings of the CSEE, 2005, 25(5): 38-43.

17. LI Yong, LIU Junyong, LIU Xiaoyu, et al. Vulnerability Assessment in Power Grid Cascading Failures Based on Entropy of Power Flow[J]. Automation of Electric Power Systems, 2012, 36(19): 11-15.

18. Shannon C E. A mathematical Theory of Communication[J]. American Telephone and Telegraph Co.], 1948, 27: 379-433.

19. JIN Bingjie, ZHANG Buhan, WANG Ke. Entropy Theory Based Optimal Power Flow Balancing Analysis in Power System[J]. Automation of Electric Power Systems, 2016, 40(12): 80-86.

20. Ou Y, Singh C. Assessment of Available Transfer Capability and Margins[J]. IEEE Power Engineering Review, 2002, 22(5): 69-69. 
21. Gravener M H, Nwankpa C. Available Transfer Capability and First Order Sensitivity[J]. IEEE Transactions on Power Systems, 1999, 14(2): $512-$ 518. 